\title{
The Ramsey number of the Fano plane versus the tight path
}

\author{
József Balogh * Felix Christian Clemen \\ Department of Mathematics \\ University of Illinois at Urbana-Champaign \\ Urbana, Illinois 61801, U.S.A. \\ jobal@math.uiuc.edu \\ fclemen2@illinois.edu \\ Jozef Skokan \\ Department of Mathematics \\ London School of Economics \\ London, U.K. \\ j.skokan@lse.ac.uk \\ Adam Zsolt Wagner ${ }^{\dagger}$ \\ Department of Mathematics \\ ETH \\ Zürich, Switzerland \\ zsolt.wagner@math.ethz.ch
}

Submitted: Dec 7, 2018; Accepted: Oct 13, 2019; Published: Mar 20, 2020

(c) The authors. Released under the CC BY license (International 4.0).

\begin{abstract}
The hypergraph Ramsey number of two 3-uniform hypergraphs $G$ and $H$, denoted by $R(G, H)$, is the least integer $N$ such that every red-blue edge-coloring of the complete 3 -uniform hypergraph on $N$ vertices contains a red copy of $G$ or a blue copy of $H$.

The Fano plane $\mathbb{F}$ is the unique 3 -uniform hypergraph with seven edges on seven vertices in which every pair of vertices is contained in a unique edge. There is a simple construction showing that $R(G, \mathbb{F}) \geqslant 2(v(G)-1)+1$ for every connected $G$. Hypergraphs $G$ for which the equality $R(G, \mathbb{F})=2(v(G)-1)+1$ holds are called $\mathbb{F}$-good. Conlon posed the problem to determine all $G$ that are $\mathbb{F}$-good.

In this short paper we make progress on this problem by proving that the tight path of length $n$ is $\mathbb{F}$-good.
\end{abstract}

Mathematics Subject Classifications: 05D10, 05C65

*and Moscow Institute of Physics and Technology, 9 Institutskiy per., Dolgoprodny, Moscow Region,141701, Russian Federation. Research is partially supported by NSF Grant DMS-1500121, Arnold O. Beckman Research Award (UIUC Campus Research Board RB 18132) and the Langan Scholar Fund (UIUC).

${ }^{\dagger}$ Research has been partially performed while at the University of Illinois at Urbana-Champaign. 


\section{Introduction}

Ramsey theory is one of the most intensively studied topics in combinatorics. Given two $k$-uniform hypergraphs $G$ and $H$, we denote by $R(G, H)$ the hypergraph Ramsey number of $G$ and $H$. That is, $R(G, H)$ is the least integer such that any red-blue edge-coloring of the complete $k$-uniform hypergraph on that many vertices contains a red $G$ or a blue $H$ as a subhypergraph. The existence of $R(G, H)$ is guaranteed by Ramsey's theorem [15]. Given a bounded degree $k$-uniform hypergraph $H$, it is known that the Ramsey number $R(H, H)$ is linear in the number of vertices of $H[4,6,7,10,12]$. However, estimating or even determining Ramsey numbers precisely is often a difficult problem.

In this short paper we will determine exactly the Ramsey number of the tight path and the Fano plane. This result is the first progress on a question asked at the AIMS workshop on hypergraph Ramsey problems in 2015 by Conlon [16]. A simple construction by Burr [1] shows that

$$
R(G, H) \geqslant(\chi(H)-1)(v(G)-1)+\sigma(H),
$$

provided $H$ is connected and $v(G) \geqslant \sigma(H)$, where $\chi(H)$ is the chromatic number of $H$ and $\sigma(H)$ is the size of the smallest color class in any $\chi(H)$-coloring of $H$. Following Burr and Erdös [1,2], we will say that $G$ is $H$-good, if (1) holds with equality. The intuition behind this definition was that $H$-good graphs tend to be poor expanders (see $[5$, Section 2.5] for further details). Denote by $\mathbb{F}$ the Fano plane, i.e. the unique 3-uniform hypergraph with seven edges on seven vertices in which every pair of vertices is contained in a unique edge. Conlon asked which hypergraphs are $\mathbb{F}$-good [16].

In the graph case, there are many exact Ramsey numbers known. Erdős [2] started the systematic study of cliques versus large graphs. Nikiforov and Rousseau [13] gave a new approach to provide exact result for several families of graphs. Recently, the Ramsey number of the cycle and the clique (Keevash, Long and Skokan [11]), and the Ramsey number of the clique and the hypercube (Griffiths, Morris, Fiz Pontiveros, Saxton, Skokan [8]) have been determined. For similar results we refer the interested reader to the excellent recent survey [5].

In the hypergraph case there are only few instances where the Ramsey number is known exactly. Our result is the first Ramsey-goodness-result for hypergraphs.

From now on, we consider only 3-uniform hypergraphs. Let $P_{n}^{t}$ be the tight path on $n$ vertices, i.e. it contains distinct vertices $v_{1}, v_{2}, \ldots, v_{n}$ and edges $e_{1}, e_{2}, \ldots, e_{n-2}$ where $e_{i}=\left\{v_{i}, v_{i+1}, v_{i+2}\right\}$.

Theorem 1. There exists $n_{0} \in \mathbb{N}$ such that for any $n \geqslant n_{0}$, we have $R\left(P_{n}^{t}, \mathbb{F}\right)=2 n-1$.

Using the definitions from above, Theorem 1 states that $P_{n}^{t}$ is $\mathbb{F}$-good. The lower bound $R\left(P_{n}^{t}, \mathbb{F}\right) \geqslant 2 n-1$ follows easily by the following folklore construction. Write the vertex set of the complete 3 -uniform hypergraph on $2 n-2$ vertices $K_{2 n-2}^{(3)}$ as the disjoint union of two sets $A$ and $B$ with $|A|=|B|=n-1$. Color all 3-edges that are fully contained in either $A$ or in $B$ red, and all other edges blue. Observe that as $|A|,|B|<n$ 
there is no red $P_{n}^{t}$ in this coloring. Since the chromatic number ${ }^{1} \chi(\mathbb{F})=3$, in every copy of $\mathbb{F}$ there is one edge that is fully contained in either $A$ or $B$, hence this coloring cannot contain a blue copy of $\mathbb{F}$ either. This establishes that $R\left(P_{n}^{t}, \mathbb{F}\right)>2 n-2$.

There is another almost extremal example, which is very different. For simplicity, let $n$ be divisible by 6 . Write the vertex set of the complete 3 -uniform hypergraph on $2 n-3$ vertices $K_{2 n-3}^{(3)}$ as the disjoint union of three sets $A, B$ and $C$ with $|A|=|B|=|C|=2 n / 3-1$. Color red all triples of vertices $\{x, y, z\}$ such that either $x, y \in A$ and $z \in A \cup B$, or $x, y \in B$ and $z \in B \cup C$, or $x, y \in C$ and $z \in A \cup C$. Color all other triples blue. A short case analysis (see Lemma 4) shows that there is no blue Fano plane $\mathbb{F}$. The longest red tight path is obtained by alternating between taking two vertices from one of the sets and one vertex from another. Such a path can have length at most $|A|+|B| / 2+1 \leqslant n-1$. The main contribution of our work is to establish the upper bound $R\left(P_{n}^{t}, \mathbb{F}\right) \leqslant 2 n-1$. In the proof of the upper bound, we will build up a picture of what a potentially bad coloring could look like and quickly realize that a bad coloring needs to be close to one of the two previous described colorings. In the remainder of the proof we then rule out these two types separately.

We remark that this proof technique also works for proving that the tight cycle is $\mathbb{F}$ good. Let $C_{n}^{(3)}$ be the tight cycle on $n$ vertices, i.e. it contains distinct vertices $v_{1}, v_{2}, \ldots, v_{n}$ and edges $e_{1}, e_{2}, \ldots, e_{n}$ with $e_{i}=\left\{v_{i}, v_{i+1}, v_{i+2}\right\}$ where $v_{n+1}:=v_{1}$ and $v_{n+2}:=v_{2}$.

Theorem 2. There exists $n_{0} \in \mathbb{N}$ such that for any $n \geqslant n_{0}$, we have $R\left(C_{n}^{(3)}, \mathbb{F}\right)=2 n-1$.

We choose not to present the proof of Theorem 2 since the proof is almost the same as the proof of Theorem 1 and differs only in some technicalities which do not give the reader more insight into the methods used.

The organization of the paper is as follows. In Section 2 we will show that Theorem 1 is sharp in the sense that the Ramsey number increases when one adds a small number of edges to the tight path. In Section 3 we will give some definitions and basic tools which will be needed for the proof of the upper bound in Theorem 1. The proof itself will be given in Section 4.

\section{Sharpness example}

The following example shows that Theorem 1 is best possible in the following sense: Let $P^{\prime}$ be the 3-uniform hypergraph obtained from the tight path $P_{n}^{t}$ by adding three edges $\left\{v_{2}, v_{3}, v_{6}\right\},\left\{v_{1}, v_{2}, v_{5}\right\}$ and $\left\{v_{1}, v_{4}, v_{6}\right\}$. We claim that Ramsey number of $P^{\prime}$ and $\mathbb{F}$ is bigger than the Ramsey number of $P_{n}^{t}$ and $\mathbb{F}$.

Theorem 3. $R\left(P^{\prime}, \mathbb{F}\right)>2 n$.

Assume, for simplicity, that $n$ is divisible by 3 . Take three sets $A, B, C$ of size $2 n / 3$ each. Color red all triples of vertices $\{x, y, z\}$ such that either $x, y \in A$ and $z \in A \cup B$, or

\footnotetext{
${ }^{1}$ Here, the chromatic number $\chi(H)$ of a hypergraph $H$ is the smallest number $k$ of colors for which there exists a $k$-coloring of the vertices of $H$ with no monochromatic edge. This is sometimes called the weak chromatic number of $H$.
} 
$x, y \in B$ and $z \in B \cup C$, or $x, y \in C$ and $z \in A \cup C$. All other triples are colored blue.

The following two Lemmas check that there is no blue $\mathbb{F}$ and no red $P_{n}^{t}$.

Lemma 4. The coloring described above does not contain a blue $\mathbb{F}$.

Proof. Let $F$ be a blue Fano plane in the previously described coloring. For $i, j, k \in[7]$ with $i+j+k=7$, we say that a Fano plane is of type $(i, j, k)$ if $i$ vertices come from $A, j$ vertices come from $B$ and $k$ vertices come from $C$. Let $a_{1}, \ldots, a_{i}$ be the vertices in $A ; b_{1}, \ldots, b_{j}$ be the vertices in $B$; and $c_{1}, \ldots, c_{k}$ the vertices in $C$. Since the chromatic number of the Fano plane is three, $F$ needs to have one vertex from each set. Thus, $F$ has to be of type $(5,1,1),(4,2,1),(4,1,2),(3,2,2)$ or $(3,3,1)$ up to rotation. Since every 5 -subset of vertices in a Fano plane contains an edge, $F$ cannot be of type $(5,1,1)$. If $F$ is of type $(4,2,1)$, then $b_{1} b_{2} c_{1}$ forms an edge or there is an edge inside $A$, because $F$ has chromatic number three. However, by construction of the coloring both of these edge are red. If $F$ is of type $(4,1,2)$, then again $c_{1} c_{2} b_{1}$ forms an edge or there is an edge inside $A$. Since the edge inside $A$ is red, $c_{1} c_{2} b_{1}$ forms an edge. In a Fano plane every pair is in exactly one edge, thus $b_{1}$ cannot be in any further blue edge. This contradicts that every vertex is in three edges. Now, let $F$ be of type $(3,2,2)$. There has to be an edge inside $\left\{b_{1}, b_{2}, c_{1}, c_{2}\right\}$. Without loss of generality let this edge be $c_{1} c_{2} b_{1}$. Now, $b_{1}$ can only be in one further edge (one containing $b_{2}$ ). This again contradicts that every vertex is in three edges. Finally, let $F$ be of type $(3,3,1)$. Again, there has to be an edge inside $\left\{b_{1}, b_{2}, b_{3}, c_{1}\right\}$. Without loss of generality let this edge be $b_{1} b_{2} c_{1}$. Now, $c_{1}$ can only be in one further edge (one containing $b_{3}$ ), contradicting that $c_{1}$ needs to have degree three.

Lemma 5. The previously described coloring does not contain a red $P_{n}^{t}$.

Proof. Let us assume there is an embedding of a red $P^{\prime}$. The first three vertices of such an embedding cannot come from different sets $A, B$ and $C$. Without loss of generality, let $A$ be the set which contains at least two of them. The only way to embed a red copy of $P_{n}^{t}$ is to use all vertices of $A$ and $n / 3$ vertices of $B$. Since between 2 vertices from $B$ there has to be at least 2 vertices from $A$, the only way for an embedding of $P_{n}^{t}$ to start is with the first 6 vertices having the following patterns: $A A B A A B, A B A A B A, A B A A A B, B A A B A A$, $B A A A B A$ or $B A A A A B$. However, regardless of which pattern we use, the resulting red tight path cannot be extended to a red copy of $P^{\prime}$ : one of $\left\{v_{2}, v_{3}, v_{6}\right\},\left\{v_{1}, v_{2}, v_{5}\right\}$ and $\left\{v_{1}, v_{4}, v_{6}\right\}$ would be of the form $B B A$ and therefore blue.

\section{Preparations}

Let the hyperedges of $\mathcal{H}:=K_{2 n-1}^{(3)}$ be two-colored with colors red and blue, without a blue $\mathbb{F}$. In the proof we will build up a picture of how this bad coloring could potentially look like over a sequence of Lemmas and eventually rule out its existence entirely.

Our starting point in the proof of Theorem 1 will be an upper bound on the off-diagonal hypergraph Ramsey numbers. We choose to use an upper bound from [3], but any weaker bound would suffice. 
Theorem 6 (Conlon-Fox-Sudakov [3]). There exists $C>0$ so that for every integer $s \geqslant 4$ and sufficiently large $t$,

$$
R\left(K_{s}^{(3)}, K_{t}^{(3)}\right) \leqslant 2^{C t^{s-2} \log t}
$$

In the proof we will make use of the following definitions.

Definition 7. Given two disjoint sets $A, B$ of vertices in $\mathcal{H}$, we say four vertices $a_{1}, a_{2} \in A$, $b_{1}, b_{2} \in B$ form a red butterfly if there exists $i, j \in\{1,2\}$ such that the two hyperedges $a_{1} a_{2} b_{i}, a_{j} b_{1} b_{2}$ are red.

We denote with $|\overrightarrow{A B}|_{r}\left(|\overrightarrow{A B}|_{b}\right)$ the number of red (blue) hyperedges in $\mathcal{H}$ of the form $a b_{1} b_{2}$ with $a \in A, b_{1}, b_{2} \in B$. Given three disjoint sets $A, B, C$ of vertices in $\mathcal{H}$, we denote with $|A B C|_{r}\left(|A B C|_{b}\right)$ the number of red (blue) hyperedges of the form $a b c$ with $a \in A, b \in B, c \in C$.

For $W \subset V(\mathcal{H}), v \notin W$, denote $G_{v, W}^{b l u e}$ the blue link graph of $v$ in $W$, i.e. the graph on $W$ with $a b$ being an edge iff $a b v$ is blue in $\mathcal{H}$. Analogously, $G_{v, W}^{r e d}$ defines the red link graph.

For $t \in \mathbb{N}$, we define the complete directed bipartite graph $\vec{K}_{t, t}$ to be the directed graph on vertex set $A \cup B$ with $|A|=|B|=t, A$ and $B$ disjoint, and the arc set $\{a b \mid a \in A, b \in B\}$.

The following theorem is a directed version of the Kövári-Sós-Turán Theorem [14].

Theorem 8. Let $t, m \in \mathbb{N}$. Define $D$ to be a digraph with vertex set $A \cup B$, where $A$ and $B$ are disjoint, and $|A|=|B|=m$. If the number of arcs from $A$ to $B$ is at least $C^{\prime} m^{2-1 / t}$ for $C^{\prime}$ being a constant large enough only depending on $t$, then $D$ contains a directed $\vec{K}_{t, t}$ from $A$ to $B$.

The following tools consisting of the next two Lemmas will be used multiple times in the main proof.

Lemma 9. Let the hyperedges of $\mathcal{H}:=K_{2 n-1}^{(3)}$ be two-colored with colors red and blue, without a blue $\mathbb{F}$. Further, let $m \in \mathbb{N}$ be big enough and $A, B, C \subseteq V(\mathcal{H})$ be disjoint sets such that $|A|=|B|=|C|=m$. Assume that there are at most 1000 vertex-disjoint red butterflies connecting each pair of the three sets $A, B, C$.

Then there exists an absolute constant $t>0$ such that

$$
|\overrightarrow{A B}|_{r},|\overrightarrow{B C}|_{r},|\overrightarrow{C A}|_{r} \leqslant m^{3-1 / t} \quad \text { or } \quad|\overrightarrow{B A}|_{r},|\overrightarrow{C B}|_{r},|\overrightarrow{A C}|_{r} \leqslant m^{3-1 / t}
$$

Proof. Removing at most 4000 vertices from each set, we end up with sets $A_{1} \subset A, A_{2} \subset$ $B, A_{3} \subset C$ so that there are no red butterflies connecting them. Note that if two vertices $a \in A_{i}$ and $b \in A_{j}$ are not contained in any red butterfly, then either all hyperedges $\left\{a b x: x \in A_{i}\right\}$ or all hyperedges $\left\{a b y: y \in A_{j}\right\}$ are blue.

Create a digraph $\vec{G}$ with $V(\vec{G})=A_{1} \cup A_{2} \cup A_{3}$ as follows. We have $\overrightarrow{u v} \in E(\vec{G})$ for $u \in A_{i}, v \in A_{j}$ with $i \neq j$ if the set of hyperedges $\left\{u v y: y \in A_{j}\right\}$ is entirely blue. Note that some edges might be oriented in both ways. 
Let $t$ be the bipartite Ramsey number for $K_{4,4}$. That is, $t$ is the least integer such that every two-coloring of the edges of $K_{t, t}$ contains a monochromatic copy of $K_{4,4}$. Note that by Irving [9], we have $t \leqslant 48$.

Suppose between $A_{1}$ and $A_{2}$, both the left and right density is at least $C^{\prime} m^{-1 / t}$ for $C^{\prime}$ being a constant large enough. By Theorem 8 we can find complete directed $\vec{K}_{t, t}$ 's in both directions. That is, there are sets $D_{1}, D_{2} \subset A_{1}$ and $E_{1}, E_{2} \subset A_{2}$ of sizes $t$ each such that the edges $\left\{\overrightarrow{a b}: a \in D_{1}, b \in E_{1}\right\}$ and $\left\{\overrightarrow{b a}: a \in D_{2}, b \in E_{2}\right\}$ are all present in $\vec{G}$.

Let $x \in D_{1}$ and set $N_{x}:=N^{+}(x) \cap A_{3}$, where $N^{+}(x)$ denotes the out-neighborhood of $x$ in $\vec{G}$. We claim $\left|N_{x}\right|<t$. Suppose towards contradiction that $\left|N_{x}\right| \geqslant t$. Since $\left|N_{x}\right| \geqslant t$ and $\left|E_{1}\right| \geqslant t$, there is a directed $\vec{K}_{4,4}$ between $N_{x}$ and $E_{1}$ in $\vec{G}$. That is, we can find $S=\left\{s_{1}, \ldots, s_{4}\right\} \subset N_{x}$ and $T=\left\{t_{1}, \ldots, t_{4}\right\} \subseteq E_{1}$ such that all edges of the form $\{\overrightarrow{s t}: s \in S, t \in T\}$ or all edges of the form $\{\overrightarrow{t s}: s \in S, t \in T\}$ are present in $\vec{G}$. Without loss of generality let all edges of the form $\{\overrightarrow{s t}: s \in S, t \in T\}$ be present. However, this is impossible as the hyperedges $\left\{x s_{1} s_{2}, x t_{1} t_{2}, x t_{3} t_{4}, s_{1} t_{1} t_{4}, s_{1} t_{2} t_{3}, s_{2} t_{1} t_{3}, s_{2} t_{2} t_{4}\right\}$ form a blue Fano plane in $\mathcal{H}$. Thus we conclude $\left|N_{x}\right|<t$.

Since $x$ was an arbitrary vertex in $D_{1}$, every vertex in $D_{1}$ has an out-neighbourhood in $A_{3}$ of size at most $t$. Repeating the same argument after replacing $D_{1}$ by $E_{2}$, we get that every vertex in $E_{2}$ has an out-neighbourhood in $A_{3}$ of size at most $t$. So by removing at most $2 t^{2}$ vertices from $A_{3}$ we get a set $A_{3}^{\prime}$ of the property that all edges from $A_{3}^{\prime}$ to $D_{1}$ and all edges from $A_{3}^{\prime}$ to $E_{2}$ are present in $\vec{G}$. By the choice of $t$, we can find sets $W_{1} \subset D_{1}$ and $W_{2} \subset E_{2}$ of sizes at least four such that without loss of generality $\left(W_{1}, W_{2}\right)$ forms a directed $K_{4,4}$. Hence we have found a 4-blowup of a transitive triangle. But this is impossible, as letting $W_{1}=\left\{v_{1}, v_{2}, v_{3}, v_{4}\right\}, W_{2}=\left\{w_{1}, w_{2}, w_{3}, w_{4}\right\}, a \in A_{3}^{\prime}$ the hyperedges $\left\{a v_{1} v_{2}, a w_{1} w_{2}, a w_{3} w_{4}, v_{1} w_{1} w_{4}, v_{1} w_{2} w_{3}, v_{2} w_{1} w_{3}, v_{2} w_{2} w_{4}\right\}$ are all blue in $\mathcal{H}$ and form a Fano plane.

This proves that in $\vec{G}$ between $A_{1}$ and $A_{2}$, in one of the directions the density has to be less than $C^{\prime} m^{-1 / t}$. Repeating this argument for the other two pairs, we get that between any pair of sets from $A_{1}, A_{2}, A_{3}$, in one of the directions the density has to be less than $C^{\prime} m^{-1 / t}$ whereas the density in the other direction has to be at least $1-C^{\prime} m^{-1 / t}$. The majority orientation forms a transitive triangle or an oriented 3-cycle. Suppose now that the majority orientation forms a transitive triangle. Pick four vertices from each set at random. Then the probability that the 12 vertices do not form a 4-blowup of the transitive triangle is at most $48 C^{\prime} m^{-t}$. Therefore, there exists a 4-blowup of a transitive triangle in $\vec{G}$, giving a blue Fano plane in $\mathcal{H}$. Thus, the majority orientation has to form a 3-cycle.

Without loss of generality let $A_{1} \rightarrow A_{2} \rightarrow A_{3} \rightarrow A_{1}$ be the majority orientation in $\vec{G}$. Then the density between $A_{1}$ and $A_{2}$ is at least $1-C^{\prime} m^{-1 / t}$ in $\vec{G}$, then this implies $\left|\overrightarrow{A_{1} A_{2}}\right|_{r} \leqslant C^{\prime} m^{3-1 / t}$ and as we only deleted at most 12000 vertices in the beginning, also $|\overrightarrow{A B}|_{r} \leqslant 2 C^{\prime} m^{3-1 / t}$. Repeating this argument for the other pairs gives us $|\overrightarrow{B C}|_{r} \leqslant$ $2 C^{\prime} m^{3-1 / t}$ and $|\overrightarrow{C A}|_{r} \leqslant 2 C^{\prime} m^{3-1 / t}$. By choosing $t$ slightly bigger we get rid of the constant $2 C^{\prime}$ for large enough $m$. 
Lemma 9 can be improved in the following way.

Lemma 10. Let the hyperedges of $\mathcal{H}:=K_{2 n-1}^{(3)}$ be two-colored with colors red and blue, without a blue $\mathbb{F}$. Further, let $m \in \mathbb{N}$ be big enough and $A, B, C \subseteq V(\mathcal{H})$ be disjoint sets such that $|A|=|B|=|C|=m$. Assume that there are at most 1000 vertex-disjoint red butterflies connecting each pair of the three sets $A, B, C$.

Then there exists an absolute constant $t>0$ such that

$$
|\overrightarrow{A B}|_{r},|\overrightarrow{B C}|_{r},|\overrightarrow{C A}|_{r},|\overrightarrow{B A}|_{b},|\overrightarrow{C B}|_{b},|\overrightarrow{A C}|_{b} \leqslant m^{3-1 / t}
$$

or

$$
|\overrightarrow{B A}|_{r},|\overrightarrow{C B}|_{r},|\overrightarrow{A C}|_{r},|\overrightarrow{A B}|_{b},|\overrightarrow{B C}|_{b},|\overrightarrow{C A}|_{b} \leqslant m^{3-1 / t}
$$

Proof. Applying Lemma 9, we get a positive constant $t^{\prime}$ such that w.l.o.g.

$$
|\overrightarrow{A B}|_{r},|\overrightarrow{B C}|_{r},|\overrightarrow{C A}|_{r} \leqslant m^{3-1 / t^{\prime}} .
$$

Let $t=3 t^{\prime}$. For the sake of contradiction, say that $|\overrightarrow{A C}|_{b} \geqslant m^{3-1 / t}$. Define

$$
Z_{1}:=\left\{v \in A \mid e\left(G_{v, B}^{b l u e}\right) \geqslant \frac{99}{100}\left(\begin{array}{c}
m \\
2
\end{array}\right)\right\} \quad \text { and } \quad Z_{1}^{\prime}:=\left\{v \in A \mid e\left(G_{v, C}^{b l u e}\right) \geqslant \frac{1}{2} m^{2-1 / t}\right\} .
$$

Then $\left|A \backslash Z_{1}\right| \leqslant 600 m^{1-1 / t^{\prime}}$, as otherwise $|\overrightarrow{A B}|_{r} \geqslant 600 m^{1-1 / t^{\prime}} \frac{1}{100}\left(\begin{array}{c}m \\ 2\end{array}\right)>2 m^{3-1 / t^{\prime}}$. Also $\left|Z_{1}^{\prime}\right| \geqslant 800 m^{1-1 / t^{\prime}}$, as otherwise $|\overrightarrow{A C}|_{b}<800 m^{1-1 / t^{\prime}} m^{2}+\frac{1}{2} m^{2-1 / t} m \leqslant m^{3-1 / t}$. Thus, one can choose a vertex $v \in Z_{1} \cap Z_{1}^{\prime}$. Let

$$
Y_{1}:=\left\{w \in B \mid e\left(G_{w, C, r}\right) \leqslant 10 m^{2-1 / t^{\prime}}\right\} .
$$

Then $\left|Y_{1}\right| \geqslant 4 / 5 m$ as otherwise $|\overrightarrow{B C}|_{r} \geqslant 2 m^{3-1 / t^{\prime}}$. Because of the size of $Y_{1}, G_{v, B}^{b l u e}$ has to contain an edge inside $Y_{1}$. Let $w_{1} w_{2}$ be such an edge. The number of 4-tuples $(a, b, c, d)$ of distinct vertices $a, b, c, d \in C$ with $a b, c d \in E\left(G_{v, C}^{b l u e}\right)$ is at least

$$
\sum_{a b \in E\left(G_{v, C}^{\text {blue }}\right)}\left(e\left(G_{v, C}^{\text {blue }}\right)-\operatorname{deg}(a)-\operatorname{deg}(b)\right) \geqslant e\left(G_{v, C}^{\text {blue }}\right)\left(e\left(G_{v, C}^{b l u e}\right)-2 m\right) \geqslant \frac{1}{5} m^{4-2 / t}
$$

The number of 4-tuples $(a, b, c, d)$ of distinct vertices $a, b, c, d \in C$ with $a d \notin E\left(G_{w_{1}, C}^{b l u e}\right)$ or $b c \notin E\left(G_{w_{1}, C}^{b l u e}\right)$ is at most $e\left(G_{w_{1}, C}^{r e d}\right) m^{2}+m^{2} e\left(G_{w_{1}, C}^{r e d}\right) \leqslant 20 m^{4-1 / t^{\prime}}$. Similarly, the number of 4-tuple $(a, b, c, d)$ of distinct vertices $a, b, c, d \in C$ with $a c \notin E\left(G_{w_{2}, C}^{b l u e}\right)$ or $b d \notin E\left(G_{w_{2}, C}^{b l u e}\right)$ is at most $20 m^{4-1 / t^{\prime}}$. Since $20 m^{4-1 / t^{\prime}}+20 m^{4-1 / t^{\prime}}<\frac{1}{5} m^{4-2 / t}$, there exists $a, b, c, d \in C$ such that $a b, c d \in E\left(G_{v, C}^{b l u e}\right) ; a d, b c \in E\left(G_{w_{1}, C}^{b l u e}\right)$ and $a c, b d \in E\left(G_{w_{2}, C}^{b l u e}\right)$. Thus, the hyperedges $v w_{1} w_{2}, v a b, v c d, w_{1} a d, w_{1} b c, w_{2} a c, w_{2} b d$ form a blue Fano plane; a contradiction, therefore we conclude that $|\overrightarrow{A C}|_{b} \leqslant m^{3-1 / t}$. Similarly, we get $|\overrightarrow{C B}|_{b} \leqslant m^{3-1 / t}$ and $|\overrightarrow{B A}|_{b} \leqslant m^{3-1 / t}$. 


\section{Proof of Theorem 1}

\subsection{Set up of the proof}

For the sake of contradiction, assume that there is a red-blue edge-coloring of $\mathcal{H}:=K_{2 n-1}^{(3)}$ without a blue $\mathbb{F}$ and without a red $P_{n}^{t}$. Fix such a coloring. Let $\varepsilon>0$ be a sufficiently small constant and assume that $n$ is sufficiently large. Set

$$
m=\left\lceil\varepsilon \sqrt[5]{\frac{\log n}{\log \log n}}\right\rceil .
$$

Observe that $m^{5} \log m \leqslant \frac{\varepsilon^{5}}{5} \log n$, hence we have by Theorem 6

$$
R\left(K_{m}^{(3)}, K_{7}^{(3)}\right) \leqslant 2^{C m^{5} \log m} \leqslant n^{\varepsilon^{4}} .
$$

Since $\mathcal{H}$ contains no blue $\mathbb{F}$, it cannot contain a blue $K_{7}^{(3)}$ and we conclude that it contains a red $K_{m}^{(3)}$, call it $D_{1}$. Set $\mathcal{H}_{1}:=\mathcal{H} \backslash V\left(D_{1}\right)$ and find a red $K_{m}^{(3)}$, call it $D_{2}$, in $\mathcal{H}_{1}$. Repeating this process, setting $\mathcal{H}_{i+1}:=\mathcal{H}_{i} \backslash V\left(D_{i}\right)$, we can find a red copy of $K_{m}^{(3)}$ in $\mathcal{H}_{i+1}$, calling it $D_{i+1}$, as long as $\left|V\left(\mathcal{H}_{i}\right)\right| \geqslant n^{\varepsilon^{4}}$. At the end of this process we end up with a collection of vertex-disjoint red $K_{m}^{(3)}$-s $D_{1}, D_{2}, \ldots, D_{d}$, and a set $J$ of remaining vertices with $|J| \leqslant n^{\varepsilon^{4}}$.

Create a graph $G_{1}$ with $V\left(G_{1}\right)=\left\{D_{1}, \ldots, D_{d}\right\}$, by connecting $D_{i}, D_{j}$ if in $\mathcal{H}$ there are at least 1000 vertex-disjoint red butterflies between them. The vertices of $G_{1}$ will be called blobs. The next two lemmas give information on the structure of $G_{1}$.

Lemma 11. The complement of $G_{1}$ contains no $K_{4}$.

Proof. For the sake of contradiction, assume that there are 4 blobs $A_{1}, A_{2}, A_{3}$ and $A_{4}$ which form a $K_{4}$ in the complement of $G_{1}$. Define a directed graph $D$ with vertex set $V(D)=\left\{A_{1}, A_{2}, A_{3}, A_{4}\right\}$ and an edge from blob $A_{i}$ to $A_{j}(i \neq j)$ iff $\left|\overrightarrow{A_{i} A_{j}}\right|_{r} \leqslant m^{3-1 / t}$ with $t$ from Lemma 10. Applying Lemma 10 on all subsets of size 3 of the 4 blobs gives that every edge in $D$ is oriented in exactly one direction. This means that $D$ is a tournament. However, a tournament on 4 vertices contains a transitive triangle and Lemma 10 says this cannot happen.

Lemma 12. $G_{1}$ has one of the following forms:

(i) $V\left(G_{1}\right)=\left\{A_{1}, \ldots, A_{a}, B_{1}, \ldots, B_{b}\right\}$ such that $A_{1}, \ldots, A_{a}$ and $B_{1}, \ldots, B_{b}$ form vertexdisjoint paths or

(ii) $V\left(G_{1}\right)=\left\{A_{1}, \ldots, A_{a}, B_{1}, \ldots, B_{b}, C_{1}, \ldots, C_{c}\right\}$ such that $A_{1}, \ldots, A_{a} ; B_{1}, \ldots, B_{b}$ and $C_{1}, \ldots, C_{c}$ form vertex-disjoint paths.

Proof. Let $A_{1}, \ldots, A_{a}$ be a longest path in $G_{1}$. If $V\left(G_{1}\right)=\left\{A_{1}, \ldots, A_{a}\right\}$ then one can find a red $P_{n}^{t}$ in $\mathcal{H}$ just by jumping from red blob to red blob along the path using 
the red butterflies. Let $B_{1}, \ldots, B_{b}$ be a longest path in $G_{1}$ on the vertices $V\left(G_{1}\right) \backslash$ $\left\{A_{1}, \ldots, A_{a}\right\}$. If $V\left(G_{1}\right)=\left\{A_{1}, \ldots, A_{a}, B_{1}, \ldots, B_{b}\right\}$ we are in case (i). Otherwise we can take the longest path $C_{1}, \ldots, C_{c}$ in $V\left(G_{1}\right) \backslash\left\{A_{1}, \ldots, A_{a}, B_{1}, \ldots, B_{b}\right\}$. In this case $V\left(G_{1}\right)=$ $\left\{A_{1}, \ldots, A_{a}, B_{1}, \ldots, B_{b}, C_{1}, \ldots, C_{c}\right\}$ as otherwise any blob in $V\left(G_{1}\right) \backslash\left\{A_{1}, \ldots, A_{a}, B_{1}, \ldots\right.$, $\left.B_{b}, C_{1}, \ldots, C_{c}\right\}$ would form a $K_{4}$ in the complement of $G_{1}$ together with $A_{1}, B_{1}$ and $C_{1}$. This is not possible by Lemma 11.

In the next two Subsections the two cases from Lemma 12 will be handled separately. The strategy is to build a long red tight path using these two or three blocks.

Remark 13. For the proof of Theorem 2 one can use the fact that when a long path say starting in $A_{1}$ and ending in $A_{a}$ is found, it is clear that some of the vertices inside the block can be used to close the cycle.

\subsection{The two paths case}

In this case $G_{1}$ is the vertex-disjoint union of two paths, i.e. in $\mathcal{H}$ we have vertex-disjoint red $K_{m}$-s $\left\{A_{1}, \ldots, A_{a}, B_{1}, \ldots, B_{b}\right\}$ and a set $J$ of junk vertices with $|J| \leqslant n^{\varepsilon^{4}}$. For every $i, j$ there are at least 1000 red butterflies between $A_{i}$ and $A_{i+1}$ and also between $B_{j}$ and $B_{j+1}$. Slightly abusing notation, let $P_{1}=\cup_{i} A_{i}$ and $P_{2}=\cup_{j} B_{j}$. Note that if $\left|P_{i}\right| \geqslant n$ for some $i \in\{1,2\}$ then we can embed the tight path $P_{n}^{t}$ into $P_{i}$ just by walking through each blob and jumping from blob to blob by using the hyperedges from the red butterflies. We know that $\left|P_{1}\right|+\left|P_{2}\right|+n^{\varepsilon^{4}} \geqslant|V(\mathcal{H})|=2 n-1$. So $n-n^{\varepsilon^{4}} \leqslant\left|P_{i}\right| \leqslant n-1$ for $i=1,2$.

Definition 14. A red triple triangle between $A_{i}$ and $B_{j}$ is a set of vertices $w, x, y, z \in A_{i}$ and $v \in B_{j}$ (or $w, x, y, z \in B_{j}$ and $v \in A_{i}$ ) so that $w x v, x y v, y z v$ is red in $\mathcal{H}$.

Observe that when we have a red triple triangle between $A_{i}$ and $B_{j}$ we can find a red tight path of length $m+1$ by swallowing one additional vertex from $B_{j}$ using the red triple triangle. If there is no red triple triangle between two blobs then there also have to be few red hyperedges between the blobs.

Lemma 15. If there is no red triple triangle between $A_{i}$ and $B_{j}$, then $\left|A_{i} B_{j}\right|_{r}+\left|B_{j} A_{i}\right|_{r} \leqslant$ $20 m^{2}$.

Proof. Pick any vertex $v \in B_{j}$ and consider its red link graph in $A_{i}$. If $v$ is not in a red triple triangle, then the red link graph does not contain a path of length 3 , hence the number of edges in this link graph is at most $10 \mathrm{~m}$. So the number of red hyperedges between $B_{j}$ and $A_{i}$, assuming that there are no red triple triangles, is at most $20 \mathrm{~m}^{2}$.

Lemma 16. $V(\mathcal{H})$ can be decomposed as $V(\mathcal{H})=A \cup B \cup J^{\prime}$ with $|A|,|B| \geqslant n-n^{\varepsilon^{3}},\left|J^{\prime}\right| \leqslant$ $n^{\varepsilon^{3}}$ such that there are at most $500 n^{3} / m$ blue hyperedges inside $A$ and respectively in $B$.

Proof. Consider the bipartite graph $G_{2}$, with vertex sets $\left\{A_{1}, \ldots, A_{a}\right\}$ and $\left\{B_{1}, \ldots, B_{b}\right\}$. Connect $A_{i} B_{j}$ by an edge iff between $A_{i}$ and $B_{j}$ there is a red triple triangle in $\mathcal{H}$. Let $M$ be a largest matching in $G_{2}$. Then we can embed into $\mathcal{H}$ a tight red path of length $\left|P_{i}\right|+|M| / 2$ for some $i \in\{1,2\}$, because at least half of the triple triangles represented by 
edges from the matching have to go in the same direction. In particular since $\left|P_{i}\right| \geqslant n-n^{\varepsilon^{4}}$ for $i=1,2$ this implies $|M| \leqslant 2 n^{\varepsilon^{4}}$. Put all blobs covered by $M$ into $J$ and get a new rubbish set $J^{\prime}$. We will have $\left|J^{\prime}\right| \leqslant|J|+2|M| m \leqslant n^{\varepsilon^{3}}$ vertices.

The subgraph of $G_{2}$ on the blobs which have not been removed spans an independent set. Let $A$ be the set of vertices in $P_{1}$ which have not been removed and let $B$ be the vertices in $P_{2}$ which have not been removed. The following argument shows that for three different blobs $A_{1}^{\prime}, A_{2}^{\prime}, A_{3}^{\prime}$ from $\left\{A_{1}, \ldots, A_{a}\right\}$ which have not been removed, $\left|A_{1}^{\prime} A_{2}^{\prime} A_{3}^{\prime}\right|_{b} \leqslant 400 m^{2}$. For contradiction, assume there are more than that many blue hyperedges. Take a blob $B_{i}$ which has not been removed from $\left\{B_{1}, \ldots, B_{b}\right\}$. By Lemma 15

$$
\left|\overrightarrow{A_{1}^{\prime} B_{i}}\right|_{r},\left|\overrightarrow{A_{2}^{\prime} B_{i}}\right|_{r},\left|\overrightarrow{A_{3}^{\prime} B_{i}}\right|_{r},\left|\overrightarrow{B_{i} A_{1}^{\prime}}\right|_{r},\left|\overrightarrow{B_{i} A_{2}^{\prime}}\right|_{r},\left|\overrightarrow{B_{i} A_{3}^{\prime}}\right|_{r} \leqslant 20 m^{2}
$$

Picking at random one vertex each of $A_{1}^{\prime}, A_{2}^{\prime}$ and $A_{3}^{\prime}$, and 4 vertices from $B_{i}$, these vertices do not form a blue Fano plane with probability at most $1-400 m^{-1}+6 \cdot 50 m^{-1}$, thus, there has to exist a blue Fano plane. We conclude $\left|A_{1}^{\prime} A_{2}^{\prime} A_{3}^{\prime}\right|_{b} \leqslant 400 \mathrm{~m}^{2}$. Therefore there are at most $400 m^{2}(n / m)^{3}+m^{3}(n / m)^{2} \leqslant 500 n^{3} / m$ blue hyperedges inside $A$. Similarly, this holds for $B$.

Definition 17. Call a vertex $v \in B$ special if $e\left(G_{v, A}^{r e d}\right) \geqslant \frac{1}{5} \varepsilon^{5}\left(\begin{array}{l}n \\ 2\end{array}\right)$. Similarly, call a vertex $v \in A$ special if $e\left(G_{v, B}^{r e d}\right) \geqslant \frac{1}{5} \varepsilon^{5}\left(\begin{array}{l}n \\ 2\end{array}\right)$.

Lemma 18. Let $V(\mathcal{H})=A \cup B \cup J^{\prime}$ be the decomposition from Lemma 16. If there are at least $n m^{-1 / 20}$ special vertices in $A$ or $B$, then one can find a red $P_{n}^{t}$ in $\mathcal{H}$.

Proof. Suppose there are at least $\mathrm{nm}^{-1 / 20}$ special vertices in w.l.o.g. $B$. We now show that we can absorb enough of these special vertices from $B$ to find a tight red path of length $n$. Let $a, b, c, d \in A, v \in B$. A tuple $(c, d)$ is called reachable from $(a, b)$ if both $a b c$ and $b c d$ are red. Further, a tuple $(c, d)$ is called reachable from $(a, b)$ via $v$ if all $a b v, b v c, v c d$ are red. A tuple $(a, b)$ is called open if there exists at most $n^{2} m^{-1 / 4}$ tuples $(c, d)$ with $c, d \in A$ such that $(c, d)$ is not reachable from $(a, b)$. Define $O$ to be the set of all open tuples. As there are at most $500 n^{3} / m$ blue hyperedges inside $A,|O| \geqslant|B|(|B|-1)-n^{2} m^{-1 / 4}$. Call a tuple $(a, b)$ good for $v \in B$ if there exists at least $\varepsilon^{100} n^{2}$ tuples $(c, d), c, d \in A$ such that $(c, d)$ is reachable from $(a, b)$ via $v$. Denote $\operatorname{Good}(v)$ the set of all tuples being good for $v$. For $v \in B$ special, $|\operatorname{Good}(v)| \geqslant \varepsilon^{100} n^{2}$, because otherwise the number of $P_{4}$ 's in $G_{v, B}^{\text {blue }}$ would be at most $2 \varepsilon^{100} n^{4}$. However, since $e\left(G_{v, B}^{b l u e}\right) \geqslant \frac{1}{5} \varepsilon^{5}\left(\begin{array}{c}n \\ 2\end{array}\right)$, the number of $P_{4}$ 's in $G_{v, B}^{\text {blue }}$ is more than $2 \varepsilon^{100} n^{4}$.

We will now walk along the red hyperedges step by step adding in each step 5 vertices to the tight path. Let $v_{1}, v_{2}, \ldots$ be the special vertices in $B$. Let $\left(a_{1}, b_{1}\right) \in \operatorname{Good}\left(v_{1}\right)$ and $\left(c_{1}, d_{1}\right)$ be an open tuple such that $\left(c_{1}, d_{1}\right)$ is reachable from $\left(a_{1}, b_{1}\right)$ via $v_{1}$. We begin the walk with $a_{1}, b_{1}, v_{1}, c_{1}, d_{1}$. Now take a look at step $i$. Assume we already have defined $a_{1}, b_{1}, v_{1}, c_{1}, d_{1}, a_{2}, \ldots, a_{i-1}, b_{i-1}, v_{i-1}, c_{i-1}, d_{i-1}$ with $\left(c_{i-1}, d_{i-1}\right)$ being open. Pick $\left(a_{i}, b_{i}\right) \in \operatorname{Good}\left(v_{i}\right)$ such that $\left(a_{i}, b_{i}\right)$ is reachable from $\left(c_{i-1}, d_{i-1}\right)$. For $i \leqslant n m^{-1 / 20}$, this is possible, because

$$
\left|\operatorname{Good}\left(v_{i}\right)\right|-n^{2} m^{-1 / 4}-5 n i \geqslant \frac{\varepsilon^{100}}{2} n^{2} .
$$


Now pick $\left(c_{i}, d_{i}\right) \in O$ such that $\left(c_{i}, d_{i}\right)$ is reachable from $\left(a_{i}, b_{i}\right)$ via $v_{1}$. For $i \leqslant n m^{-1}$, this is possible, because

$$
\varepsilon^{100} n^{2}-n^{2} m^{-1 / 4}-5 n i \geqslant \frac{\varepsilon^{100}}{2} n^{2}
$$

Now enlarge the path with $a_{i} b_{i} v_{i} c_{i} d_{i}$. After $i \leqslant n m^{-1 / 20}$ steps we end up with a path of length $5 \mathrm{~nm}^{-1 / 20}$ such that the last two vertices form an open tuple. Now, we just keep picking open tuples and walk from an open tuple to an open tuple. We can keep doing this until at most $n m^{-1 / 10}$ vertices are not used inside $A$. This means we found a tight red path of length at least

$$
n-n^{\varepsilon^{4}}-n m^{-1 / 10}+n m^{-1 / 20} \geqslant n .
$$

Lemma 19. Let $V(\mathcal{H})=A \cup B \cup J^{\prime}$ be the decomposition from Lemma 16. If there are at most $n m^{-1 / 20}$ special vertices in $A$ and $B$, then the vertex set $V(\mathcal{H})$ can be decomposed into $V(\mathcal{H})=A^{\prime} \cup B^{\prime} \cup J^{\prime \prime}$ with $\left|A^{\prime}\right|,\left|B^{\prime}\right| \geqslant n-\varepsilon n,\left|J^{\prime \prime}\right| \leqslant \varepsilon n$ such that $\mathcal{H}\left[A^{\prime}\right]$ and $\mathcal{H}\left[B^{\prime}\right]$ are entirely red, for all $v \in A^{\prime} e\left(G_{v, B^{\prime}}^{r e d}\right) \leqslant \frac{1}{5} \varepsilon^{5} n^{2}$ and for all $v \in B^{\prime} e\left(G_{v, A^{\prime}}^{r e d}\right) \leqslant \frac{1}{5} \varepsilon^{5} n^{2}$.

Proof. We can remove all special vertices from $A$ and $B$ and add them to the junk set $J^{\prime}$. So we obtain $A^{\prime}, B^{\prime}, J^{\prime \prime}$ so that for each $v \in A^{\prime}$ the red link graph in $B^{\prime}$ has at most $\frac{1}{5} \varepsilon^{5} n^{2}$ edges, for each $w \in B^{\prime}$ the red link graph of $w$ in $A^{\prime}$ has at most $\frac{1}{5} \varepsilon^{5} n^{2}$ edges and $\left|J^{\prime \prime}\right| \leqslant\left|J^{\prime}\right|+2 n m^{-1 / 20} \leqslant \varepsilon n$.

Suppose $a b c$ is a blue hyperedge in $A^{\prime}$. Let $G_{a, B^{\prime}}^{b l u e}, G_{b, B^{\prime}}^{b l u e}, G_{c, B^{\prime}}^{b l u e}$ be the blue link graphs in $B^{\prime}$. By the previous observation $e\left(G_{a, B^{\prime}}^{\text {blue }} \cap G_{b, B^{\prime}}^{\text {blue }} \cap G_{c, B^{\prime}}^{\text {blue }}\right) \geqslant \frac{9}{10}\left(\begin{array}{c}n \\ 2\end{array}\right)$ and thus $G_{a, B^{\prime}}^{\text {blue }} \cap G_{b, B^{\prime}}^{\text {blue }} \cap G_{c, B^{\prime}}^{\text {blue }}$ contains a $K_{4}$. The four vertices from the $K_{4}$ together with $a, b, c$ contain a blue copy of the Fano plane in $\mathcal{H}$. Hence $\mathcal{H}\left[A^{\prime}\right]$ is entirely red. The same holds for $\mathcal{H}\left[B^{\prime}\right]$.

Lemma 20. In the setting of Lemma 19, we can decompose $J^{\prime \prime}=J_{1} \cup J_{2}$ such that for all $v \in J_{1} e\left(G_{v, A^{\prime}}^{b l u e}\right) \leqslant \varepsilon n^{2}$ and all $v \in J_{2} e\left(G_{v, B^{\prime}}^{b l u e}\right) \leqslant \varepsilon n^{2}$.

Proof. Let $V(\mathcal{H})=A^{\prime} \cup B^{\prime} \cup J^{\prime \prime}$ be the decomposition from Lemma 19. We actually will prove that if a rubbish vertex $v \in J^{\prime \prime}$ has $e\left(G_{v, A^{\prime}}^{b l u e}\right) \geqslant \varepsilon n^{2}$, then it cannot have a blue hyperedge into $B^{\prime}$. Indeed suppose there are $a, b \in B^{\prime}$ with $a b v$ blue. Then both $a$ and $b$ are part of at least $\left(\begin{array}{c}\left|A^{\prime}\right| \\ 2\end{array}\right)-\frac{1}{5} \varepsilon^{5} n^{2}$ blue hyperedges with the other two vertices being in $A^{\prime}$ by the final statement in Lemma 19. Therefore there are at least $\left(\begin{array}{c}\left|A^{\prime}\right| \\ 2\end{array}\right)-\frac{2}{5} \varepsilon^{5} n^{2}$ pairs $(c, d)$ with $c, d \in A^{\prime}$ and $c d a, c d b$ blue, call this set of edges $S$. Now suppose $v$ leads at least $\varepsilon n^{2}$ blue

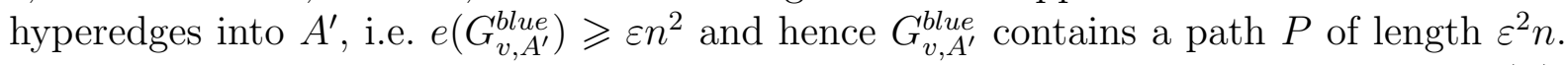
The restriction of $S$ onto the vertex set of $P=\left\{p_{1}, p_{2}, \ldots\right\}$ contains at least $(1-\varepsilon)\left(\begin{array}{c}|P| \\ 2\end{array}\right)$ edges and hence contains four vertices $p_{i}, p_{i+1}, p_{j}, p_{j+1}$ with $p_{i} p_{j}, p_{i} p_{j+1}, p_{i+1} p_{j}, p_{i+1} p_{j+1}$ in $S$. Then $a p_{i} p_{j+1}, a v b, a p_{j} p_{i+1}, p_{j+1} b p_{i+1}, p_{j+1} v p_{j}, p_{i} v p_{i+1}, p_{i} p_{j} b$ form a blue Fano plane. Hence we can split up the junk set $J^{\prime \prime}=J_{1} \cup J_{2}$ such that for all $v \in J_{1} e\left(G_{v, A^{\prime}}^{b l u e}\right) \leqslant \varepsilon n^{2}$ and all $v \in J_{2} e\left(G_{v, B^{\prime}}^{b l u e}\right) \leqslant \varepsilon n^{2}$.

Lemma 21. In the setting of Lemma 19, we can find a red $P_{n}^{t}$ in $\mathcal{H}$. 
Proof. Let $J^{\prime \prime}=J_{1} \cup J_{2}$ be the decomposition of the junk vertices from Lemma 20. Set $A^{*}=A^{\prime} \cup J_{1}$ and $B^{*}=B^{\prime} \cup J_{2}$. Then either $\left|A^{*}\right| \geqslant n$ or $\left|B^{*}\right| \geqslant n$. W.l.o.g. $\left|A^{*}\right| \geqslant n$. Now one can find a red tight path of length $n$ inside $A^{*}$. Let $v_{1}, v_{2}, \ldots$ be the vertices from $J_{1}$. Call a tuple $(a, b), a, b \in A^{\prime}$ good $^{*}$ for $v \in J_{1}$ if the red link graph of $v$ in $A^{\prime}$ contains at least $\frac{9}{10} n^{2}$ tuples $(c, d), c, d \in A^{\prime}$ such that $a b v, b v c, v c d$ are red in $\mathcal{H}$. Since the blue link graph of $v$ contains at most $\varepsilon n^{2}$ edges, for each $v \in J_{1}$ the number of good* tuples is at least $\frac{9}{10} n^{2}$. Now start with an arbitrary good* tuple $\left(a_{1}, b_{1}\right)$ for $v_{1}$. The start of the walk is $a_{1}, b_{1}, v_{1}$. Now assume we already have chosen $a_{1}, b_{1}, v_{1}, \ldots, a_{i-1}, b_{i-1}, v_{i-1}$ such that $\left(a_{i-1}, b_{i-1}\right)$ is good* for $v_{i-1}$. Take a good* tuple $\left(a_{i}, b_{i}\right)$ for $v_{i}$ of unused vertices such that $b_{i-1} v_{i-1} a_{i}, v_{i-1} a_{i} b_{i}$ are red. This is possible for all $i \leqslant \varepsilon n$, because

$$
\frac{9}{10} n^{2}-\frac{1}{10} n^{2}-3 i n>0
$$

Enlarge the path by $a_{i} b_{i} v_{i}$. After all vertices from $J_{1}$ are used, just walk through $A^{\prime}$ until all vertices in $A^{\prime}$ are used. This is possible, because all hyperedges inside $A^{\prime}$ are red. Thus, we find a red tight path of length $\left|A^{*}\right| \geqslant n$.

\subsection{The three paths case}

Let $A, B, C$ with $A=\left\{A_{1}, A_{2}, \ldots, A_{a}\right\}, B=\left\{B_{1}, \ldots, B_{b}\right\}$ and $C=\left\{C_{1}, \ldots, C_{c}\right\}$ be the three paths in $G_{1}$. There cannot be an edge between blobs of different paths, otherwise we can reduce this case to the two path case from Section 4.2 in the following way. Without loss of generality, assume that there is an edge between $A_{j}$ and $B_{k}$. Split up each blob from $A$ and $B$ into two blobs of equal size (if $m$ is odd one vertex ends up in $J$ ) in such a way that blobs coming from consecutive blobs still have at least 100 disjoint red butterflies between them and such that there are also still at least 100 disjoint red butterflies between the blobs coming from $A_{j}$ and $B_{k}$. Now, when one constructs the graph of all new blobs, where two blobs are adjacent with each other when they have at least 100 disjoint red butterflies between them, then this graph can be decomposed into two paths. We already handled this case in Subsection 4.2. Therefore we can assume that there are no edges between blobs of different paths in $G_{1}$.

Using Lemma 10 it follows that w.l.o.g. $\left|\overrightarrow{A_{i} B_{j}}\right|_{r},\left|\overrightarrow{B_{j} C_{k}}\right|,\left|\overrightarrow{C_{k} A_{i}}\right|_{r} \leqslant m^{3-1 / t}$ for all $i, j, k$. Let $P_{1}=\cup A_{i}, P_{2}=\cup B_{i}$ and $P_{3}=\cup C_{i}$. Clearly, $\left|P_{1}\right|,\left|P_{2}\right|,\left|P_{3}\right|<n$ as otherwise one could find a red tight path of length $n$ just by going through a blob and then jumping to the next by using a red butterfly and so on.

Definition 22. For $X, Y \subset V(\mathcal{H})$ and $0 \leqslant \alpha \leqslant 1$, denote $G(X, Y, \alpha)$ the graph with vertex set $X$, and $a b$ is an edge iff the number of red hyperedges $a b c$ with $c$ from $Y$ is at least $\alpha|Y|$.

Lemma 23. There exists a constant $C^{\prime}$ such that

$$
\frac{2}{3} n-C^{\prime} n m^{-1 / t} \leqslant\left|P_{l}\right| \leqslant \frac{2}{3} n+C^{\prime} n m^{-1 / t}
$$

for $l=1,2,3$. 
Proof. Let $A_{i} \in A, B_{i} \in B$. We will show that one can find a red tight path of length at least $3 \mathrm{~m} / 2-4000 \mathrm{~m}^{1-1 / t}$ just using $A_{i}$ and $B_{i}$ starting with two vertices from $A_{i}$, ending with two vertices from $A_{i}$, not using two vertices being part of a butterfly to $A_{i-1}$ and not using two other vertices being part of a butterfly to $A_{i+1}$. Consider the graph $G\left(A_{i}, B_{i}, 0.99\right)$. The number of vertices in this graph with degree at most $0.9 \mathrm{~m}$ is at most $2000 m^{1-1 / t}$ as otherwise $\left|\overrightarrow{B_{i} A_{i}}\right|_{r}>2000 m^{1-1 / t} \cdot 0.1 m \cdot 0.01 m \cdot 0.5=m^{3-1 / t}$. Let $A^{\prime} \subseteq A_{i}$ be the set of all vertices of degree at least $0.9 \mathrm{~m}$ not containing the two vertices being part of a butterfly to $A_{i-1}$ and not containing the two vertices being part of a butterfly to $A_{i+1}$. Then $\left|A^{\prime}\right| \geqslant m-2000 m^{1-1 / t}-4, G\left(A^{\prime}, B_{i}, 0.99\right)$ has minimum degree at least $0.8 \mathrm{~m}$ and thus there exists a Hamiltonian path $v_{1}, v_{2}, \ldots, v_{A^{\prime}}$ in this graph. After every second vertex in this path we will now add a vertex from $B_{i}$ to find a long red tight path in $A_{i} \cup B_{i}$. Assume we already found the tight red path $v_{1}, v_{2}, w_{1}, v_{3}, v_{4}, w_{2}, \ldots, v_{2 j-1}, v_{2 j}$. Then we can pick a vertex $w_{j} \in B_{i}$ which has not been used yet and such that $v_{2 j-1} v_{2 j} w_{j}, v_{2 j} w_{j}, v_{2 j+1}, w_{j}, v_{2 j+1} v_{2 j+2}$ are red for $j<\left|A^{\prime}\right| / 2$. This is possible because $m-0.01 m-0.01 m-0.01 m-j>0$. Thus, we can find a red tight path of length at least

$$
\frac{3}{2}\left(m-2001 m^{1-1 / t}\right) \geqslant \frac{3 m}{2}-4000 m^{1-1 / t} .
$$

If $\left|P_{1}\right| \leqslant\left|P_{2}\right|$, then we can find a tight red path of length at least $3 / 2\left|P_{1}\right|-5000 \mathrm{~nm}^{-1 / t}$ by the following argument. Jump from blob to blob in $A$ using the vertices from the butterflies and always absorbing the vertices from the index corresponding blob in $B$. When we are done with all blobs in $A$ we stop and have found a red tight path of length at least

$$
\left(\frac{3}{2} m-4000 m^{1-1 / t}\right)\left\lfloor\frac{\left|P_{1}\right|}{m}\right\rfloor \geqslant\left(\frac{3}{2} m-4000 m^{1-1 / t}\right)\left(\frac{\left|P_{1}\right|}{m}-1\right) \geqslant \frac{3}{2}\left|P_{1}\right|-5000 n m^{-1 / t} .
$$

If $\left|P_{1}\right| \geqslant\left|P_{2}\right|$, then we can find a tight red path of length at least $\left(\left|P_{1}\right|+\left|P_{2}\right| / 2\right)-$ $5000 \mathrm{~nm}^{-1 / t}$ by the following argument. Jump from blob to blob in $A$ using the vertices from the butterflies and always absorbing the vertices from the index-corresponding blob in $B$. When we are done with all blobs in $B$ we go back to $A$ and walk through the remaining blobs from $A$ using the butterflies. So we can find a tight red path of length at least

$$
\left(\frac{3}{2} m-4000 m^{1-1 / t}\right)\left\lfloor\frac{\left|P_{2}\right|}{m}\right\rfloor+\left|P_{1}\right|-\left|P_{2}\right| \geqslant\left|P_{1}\right|+\frac{\left|P_{2}\right|}{2}-5000 n m^{-1 / t} .
$$

We will now show that the sizes of the blocks $P_{1}, P_{2}, P_{3}$ is at most $2 / 3 n+C^{\prime} n m^{-1 / t}$ and at least $2 / 3 n-C^{\prime} n m^{-1 / t}$ for an absolute constant $C^{\prime}$. W.l.o.g. let $P_{1}$ be a biggest block. If $\left|P_{1}\right| \leqslant 2 / 3 n+30000 \mathrm{~nm}^{-1 / t}$, then also $\left|P_{2}\right|,\left|P_{3}\right| \leqslant 2 / 3 n+30000 \mathrm{~nm}^{-1 / t}$, but since $\left|P_{1}\right|+\left|P_{2}\right|+\left|P_{3}\right|=2 n-1-|J|$, we also get $\left|P_{1}\right|,\left|P_{2}\right|,\left|P_{3}\right| \geqslant 2 / 3 n-60001 n^{-1 / t}$. Assume $\left|P_{1}\right| \geqslant 2 / 3 n+30000 \mathrm{~nm}^{-1 / t}$. If $\left|P_{2}\right| \geqslant 2\left(n-\left|P_{1}\right|\right)+10000 \mathrm{~nm}^{-1 / t}$, then we find a red tight path of length at least

$$
\left(\left|P_{1}\right|+\frac{1}{2}\left|P_{2}\right|\right)-5000 n m^{-1 / t} \geqslant n+5000 n m^{-1 / t} .
$$


Otherwise, $\left|P_{2}\right| \leqslant 2\left(n-\left|P_{1}\right|\right)+10000 \mathrm{~nm}^{-1 / t}$. Then, $\left|P_{1}\right|+\left|P_{2}\right| \leqslant 2 n-\left|P_{1}\right|+10000 \mathrm{~nm}^{-1 / t}$ and thus $\left|P_{3}\right| \geqslant\left|P_{1}\right|-10001 \mathrm{~nm}^{-1 / t} \geqslant 2 / 3 n+19999 \mathrm{~nm}^{-1 / t}$. But now we can find a red tight path of length at least $\frac{3}{2}\left|P_{1}\right|-5000 \mathrm{~nm}^{-1 / t}>n$. This shows that there exists a constant $C^{\prime}$ such that

$$
\frac{2}{3} n-C^{\prime} n m^{-1 / t} \leqslant\left|P_{l}\right| \leqslant \frac{2}{3} n+C^{\prime} n m^{-1 / t}
$$

for $l=1,2,3$.

Lemma 24. $V(\mathcal{H})$ can be decomposed as $V(\mathcal{H})=P_{1}^{\prime \prime} \cup P_{2}^{\prime \prime} \cup P_{3}^{\prime \prime} \cup J$ such that $\left|P_{1}^{\prime \prime}\right|=$ $\left|P_{2}^{\prime \prime}\right|=\left|P_{3}^{\prime \prime}\right| ;|J| \leqslant C^{\prime \prime} n m^{-1 / t}$ for some constant $C^{\prime \prime}>0$ and each of $\mathcal{H}\left[P_{1}^{\prime \prime}\right], \mathcal{H}\left[P_{2}^{\prime \prime}\right], \mathcal{H}\left[P_{3}^{\prime \prime}\right]$ is entirely red.

Proof. The number of vertices $v \in A_{1}$ with $e\left(G_{v, B_{1}}^{\text {blue }}\right) \leqslant 29 / 30\left(\begin{array}{c}m \\ 2\end{array}\right)$ is at most $30 m^{1-1 / t}$ as otherwise $\left|\overrightarrow{A_{1} B_{1}}\right|_{r} \geqslant m^{3-1 / t}$. This means one can move at most $30 m^{1-1 / t}(n / m) \leqslant$ $30 \mathrm{~nm}^{-1 / t}$ vertices $v$ from $P_{1}$ to $J$ (and obtain $P_{1}^{\prime} \subseteq P_{1}$ ) such that all vertices in $P_{1}^{\prime}$ satisfy $e\left(G_{v, B_{1}}^{\text {blue }}\right) \geqslant 29 / 30\left(\begin{array}{c}m \\ 2\end{array}\right)$. Now assume there is a blue hyperedge $v_{1} v_{2} v_{3}$ inside $P_{1}^{\prime}$. Since $e\left(G_{v_{1}, B_{1}}^{\text {blue }} \cap G_{v_{2}, B_{1}}^{\text {blue }} \cap G_{v_{3}, B_{1}}^{\text {blue }}\right) \geqslant 27 / 30\left(\begin{array}{c}m \\ 2\end{array}\right), G_{v_{1}, B_{1}}^{\text {blue }} \cap G_{v_{2}, B_{1}}^{\text {blue }} \cap G_{v_{3}, B_{1}}^{\text {blue }}$ contains a $K_{4}$. These 4 vertices together with $v_{1}, v_{2}, v_{3}$ form a blue Fano plane. Thus $P_{1}^{\prime}$ is entirely red. Repeating this cleaning procedure for $P_{2}$ and $P_{3}$ one ends up with entire red blocks $P_{1}^{\prime}, P_{2}^{\prime}, P_{3}^{\prime}$ and a rubbish set $J^{\prime}$ of size at most $100 \mathrm{~nm}^{-1 / t}$. Considering that the blocks had roughly equal size, we can remove a few more vertices from the blocks and end up with entirely red blocks $P_{1}^{\prime \prime}, P_{2}^{\prime \prime}, P_{3}^{\prime \prime}$ of equal size and a rubbish set $J^{\prime \prime}$ of size at most $C^{\prime \prime} n m^{-1 / t}$ vertices with $C^{\prime \prime}$ being an absolute constant.

Lemma 25. Let $V(\mathcal{H})=P_{1}^{\prime \prime} \cup P_{2}^{\prime \prime} \cup P_{3}^{\prime \prime} \cup J$ be the decomposition from Lemma 24. Then $\left|P_{1}^{\prime \prime} P_{2}^{\prime \prime} P_{3}^{\prime \prime}\right|_{r} \leqslant 7 n^{3-1 / t}$.

Proof. Applying Lemma 10 gives w.l.o.g. that

$$
\left|\overrightarrow{P_{1}^{\prime \prime} P_{2}^{\prime \prime}}\right|_{r},\left|\overrightarrow{P_{2}^{\prime \prime} P_{3}^{\prime \prime \prime}}\right|_{r},\left|\overrightarrow{P_{3}^{\prime \prime} P_{1}^{\prime \prime}}\right|_{r},\left|\overrightarrow{P_{2}^{\prime \prime} P_{1}^{\prime \prime}}\right|_{b},\left|\overrightarrow{P_{3}^{\prime \prime} P_{2}^{\prime \prime \prime}}\right|_{b},\left|\overrightarrow{P_{1}^{\prime \prime} P_{3}^{\prime \prime}}\right|_{b} \leqslant n^{3-1 / t}
$$

Assume $\left|P_{1}^{\prime \prime} P_{2}^{\prime \prime} P_{3}^{\prime \prime}\right|_{r} \geqslant 7 n^{3-1 / t}$. Pick $v_{1}, w_{1} \in P_{1}^{\prime \prime}, v_{2}, w_{2}, x_{2} \in P_{2}^{\prime \prime}, v_{3} \in P_{3}^{\prime \prime}$ uniformly at random. The hyperedge $v_{1} w_{1} v_{2}$ is blue or not a proper hyperedge with probability at most $2 n^{-1 / t}$. Similarly, $v_{2} w_{2} v_{3}$ and $v_{3} w_{2} x_{2}$ is blue or not an hyperedge each with probability at most $2 n^{-1 / t}$. The hyperedge $v_{1} v_{2} v_{3}$ is blue with probability at most $1-7 n^{3-1 / t}$. Thus, the probability that one of the hyperedges $v_{1} w_{1} v_{2}, v_{1} v_{2} v_{3}, v_{2} v_{3} w_{2}, v_{3} w_{2} x_{2}$ is blue is at most $1-7 n^{3-1 / t}+6 n^{3-1 / t}<1$. Thus, there exists $v_{1}, v_{2}, v_{3}, w_{1}, w_{2}, w_{3}$ such that all the hyperedges $v_{1} w_{1} v_{2}, v_{1} v_{2} v_{3}, v_{2} v_{3} w_{2}, v_{3} w_{2} x_{2}$ are red. Now one can find a red tight path of length at least $\left|P_{1}^{\prime \prime}\right|+\left|P_{2}^{\prime \prime}\right|+1 \geqslant n$ by first going through all vertices in $P_{1}^{\prime \prime}$ besides $v_{1}$ and $w_{1}$, then going along $w_{1} v_{1} v_{2} v_{3} w_{1} w_{2}$ and then through all vertices in $P_{2}^{\prime \prime}$. Recall that all hyperedges inside $P_{1}^{\prime \prime}, P_{2}^{\prime \prime}$ or $P_{3}^{\prime \prime}$ are red.

Lemma 26. There exists a decomposition of the vertices of $\mathcal{H}$ into $V(\mathcal{H})=P_{1}^{\dagger} \cup P_{2}^{\dagger} \cup$ $P_{3}^{\dagger}$ with $0.66 n \leqslant\left|P_{l}^{\dagger}\right| \leqslant 0.67 n$ for $l=1,2,3$ such that all graphs $G\left(P_{1}^{\dagger}, P_{2}^{\dagger}, 0.98\right)$, $G\left(P_{2}^{\dagger}, P_{3}^{\dagger}, 0.98\right)$ and $G\left(P_{3}^{\dagger}, P_{1}^{\dagger}, 0.98\right)$ have minimum degree at least $0.39 n$. 
Proof. Let $V(\mathcal{H})=P_{1}^{\prime \prime} \cup P_{2}^{\prime \prime} \cup P_{3}^{\prime \prime} \cup J$ be the decomposition from Lemma 24. The number of vertices in $G\left(P_{1}^{\prime \prime}, P_{2}^{\prime \prime}, 0.99\right)$ with degree less than $0.4 n$ is at most $1500 n^{1-1 / t}$. Removing at most $1500 n^{1-1 / t}$ vertices from each $P_{1}^{\prime \prime}, P_{2}^{\prime \prime}, P_{3}^{\prime \prime}$ leaves us with sets $P_{1}^{*}, P_{2}^{*}, P_{3}^{*}$ and a junk set $J^{*}$ such that every vertex in the graphs $G\left(P_{1}^{*}, P_{2}^{*}, 0.98\right), G\left(P_{2}^{*}, P_{3}^{*}, 0.98\right)$ and $G\left(P_{3}^{*}, P_{1}^{*}, 0.98\right)$ has minimum degree at least $0.39 n$.

We now check that every vertex $v$ in $J^{*}$ has degree at least $0.39 n$ in one of the graphs $G\left(P_{1}^{*} \cup\{v\}, P_{2}^{*}, 0.98\right), G\left(P_{2}^{*} \cup\{v\}, P_{3}^{*}, 0.98\right)$ and $G\left(P_{3}^{*} \cup\{v\}, P_{1}^{*}, 0.98\right)$. Assume this is not the case, then there exists $v \in J^{*}, X_{1} \subseteq P_{1}^{*}, X_{2} \subseteq P_{2}^{*}$ and $X_{3} \subseteq P_{3}^{*}$ with $\left|X_{1}\right|=\left|X_{2}\right|=\left|X_{3}\right| \geqslant 0.65 n-0.39 n=0.26 n$ such that for each $x_{1} \in X_{1}$ there are at least $0.02 \cdot 0.65 n \geqslant 0.01 n$ many vertices $y_{2} \in P_{2}^{*}$ such that $v x_{1} y_{2}$ is blue. Similarly, for each $x_{2} \in X_{2}$ there are at least $0.01 n$ many vertices $y_{3} \in P_{3}^{*}$ such that $v x_{2} y_{3}$ is blue and for each $x_{3} \in X_{3}$ there are at least $0.01 n$ many vertices $y_{1} \in P_{1}^{*}$ such that $v x_{3} y_{1}$ is blue. Now pick $x_{1} \in X_{1}, x_{2} \in X_{2}, x_{3} \in X_{3}$ independently uniformly at random. There exist random sets $Y_{1} \subset P_{1}^{*}, Y_{2} \subset P_{2}^{*}, Y_{3} \subset P_{3}^{*}$ with $\left|Y_{1}\right|=\left|Y_{2}\right|=\left|Y_{3}\right| \geqslant 0.01 n$ such that $v x_{1} y_{2}, v x_{2} y_{3}, v x_{3} y_{1}$ for all $y_{1} \in Y_{1}, y_{2} \in Y_{2}, y_{3} \in Y_{3}$. Now pick $y_{1} \in Y_{1}, y_{2} \in Y_{2}, y_{3} \in Y_{3}$ independently uniformly at random. The hyperedges $v x_{1} y_{2}, v x_{2} y_{3}, v x_{3} y_{1}$ are blue. As $\left|X_{1} X_{2} X_{3}\right|_{r} \leqslant\left|P_{1}^{\prime \prime} P_{2}^{\prime \prime} P_{3}^{\prime \prime}\right|_{r} \leqslant n^{3-1 / t}, x_{1} x_{2} x_{3}$ is red with probability at most $4^{3} n^{-1 / t}$. Since

$$
\left|Y_{1} X_{2} Y_{2}\right|_{r} \leqslant\left|\overrightarrow{P_{1}^{\prime \prime} P_{2}^{\prime \prime}}\right|_{r} \leqslant n^{3-1 / t}, \quad\left|Y_{2} X_{3} Y_{3}\right|_{r} \leqslant\left|\overrightarrow{P_{2}^{\prime \prime} P_{3}^{\prime \prime}}\right|_{r} \leqslant n^{3-1 / t}
$$

and $\left|Y_{3} X_{1} Y_{1}\right|_{r} \leqslant\left|\overrightarrow{P_{3}^{\prime \prime} P_{1}^{\prime \prime}}\right|_{r} \leqslant n^{3-1 / t}$, the probability that each of the hyperedges $y_{1} x_{2} y_{2}$, $y_{2} x_{3} y_{3}, y_{3} x_{1} y_{1}$ is red is at most $C^{*} n^{-1 / t}$ for an absolute constant $C^{*}$. Thus, with positive probability $v x_{1} y_{2}, v x_{2} y_{3}, v x_{3} y_{1}, x_{1} x_{2} x_{3}, y_{1} x_{2} y_{2}, y_{2} x_{3} y_{3}, y_{3} x_{1} y_{1}$ form a blue Fano plane. We therefore can assume that every vertex $v \in J^{*}$ has degree at least $0.39 n$ in one of the graphs $G\left(P_{1}^{*} \cup\{v\}, P_{2}^{*}, 0.98\right), G\left(P_{2}^{*} \cup\{v\}, P_{3}^{*}, 0.98\right)$ and $G\left(P_{3}^{*} \cup\{v\}, P_{1}^{*}, 0.98\right)$. Thus every vertex from $J$ can be added to $P_{1}^{*}$ or $P_{2}^{*}$ or $P_{3}^{*}$ such that one obtains blocks $P_{1}^{\dagger}, P_{2}^{\dagger}, P_{3}^{\dagger}$ $\left(0.66 n \leqslant\left|P_{l}^{\dagger}\right| \leqslant 0.67 n\right.$ for $\left.l=1,2,3\right)$ with $P_{1}^{\dagger} \cup P_{2}^{\dagger} \cup P_{3}^{\dagger}=[2 n-1]$ in such a way that afterwards all graphs $G\left(P_{1}^{\dagger}, P_{2}^{\dagger}, 0.98\right), G\left(P_{2}^{\dagger}, P_{3}^{\dagger}, 0.98\right)$ and $G\left(P_{3}^{\dagger}, P_{1}^{\dagger}, 0.98\right)$ have minimum degree at least $0.39 n$.

Lemma 27. In the setting of Lemma 26 we can find a red $P_{n}^{t}$.

Proof. Since $P_{1}^{\dagger} \cup P_{2}^{\dagger} \cup P_{3}^{\dagger}=[2 n-1]$ one of the blocks has size at least $2 n / 3$. W.l.o.g. $\left|P_{1}^{\dagger}\right| \geqslant 2 n / 3$. The minimum degree of $G\left(P_{1}^{\dagger}, P_{2}^{\dagger}, 0.98\right)$ assures that this graph contains a Hamiltonian path. Label such a path $a_{1}, a_{2}, a_{3}, \ldots, a_{\left|P_{1}^{\dagger}\right|}$. In order to find a tight path of length $n$, we will add after every second vertex in this path a vertex from $P_{2}^{\dagger}$. Assume we already have found $a_{1}, a_{2}, b_{1}, a_{3}, a_{4}, b_{2}, \ldots, a_{2 i-1} a_{2 i}$ then we can choose $b_{i}$ from $P_{2}^{\dagger}$ which has not been used so far such that $a_{2 i-1} a_{2 i} b_{i}, a_{2 i} b_{i} a_{2 i+1}$ and $b_{i} a_{2 i+1}, a_{2 i+2}$ is red, because $\left|P_{2}^{\dagger}\right|-0.02\left|P_{2}^{\dagger}\right|-0.02\left|P_{2}^{\dagger}\right|-0.02\left|P_{2}^{\dagger}\right|-i>0$ for $i<0.94\left|P_{2}^{\dagger}\right|$ and thus especially for $i<0.5 n$. Hence, we can embed a red tight path of length at least $2 n / 3+n / 3=n$.

\section{Acknowledgement}

We would like to thank an anonymous referee for helpful comments. 


\section{References}

[1] S. A. Burr, What can we hope to accomplish in generalized Ramsey theory?, Discrete Mathematics, 67(3) (1987), 215-225.

[2] S. A. Burr, P. Erdős, Generalizations of a Ramsey-theoretic result of Chvátal, Journal of Graph Theory 7.1 (1983), 39-51.

[3] D. Conlon, J. Fox, B. Sudakov, Hypergraph Ramsey numbers, J. Amer. Math. Soc. 23 (2010), no. 1, 247-266.

[4] D. Conlon, J. Fox, B. Sudakov, Ramsey numbers of sparse hypergraphs, Random Structures \& Algorithms 35.1 (2009), 1-14.

[5] D. Conlon, J. Fox, B. Sudakov, Recent developments in graph Ramsey theory, Surveys in combinatorics 424 (2015): 49-118.

[6] O. Cooley, N. Fountoulakis, D. Kühn, D. Osthus, : Embeddings and Ramsey numbers of sparse k-uniform hypergraphs, Combinatorica 28 (2009), 263-297

[7] O. Cooley, N. Fountoulakis, D. Kühn, D. Osthus, 3-uniform hypergraphs of bounded degree have linear Ramsey numbers, J. Combinatorial Theory Ser. B 98 (2008), no. $3,484-505$.

[8] S. Griffiths, R. Morris, G. Fiz Pontiveros, D. Saxton, J. Skokan, The Ramsey number of the clique and the hypercube, J. Lond. Math. Soc. 89 (2014), 680-702.

[9] R. W. Irving, A bipartite Ramsey problem and the Zarankiewicz numbers, Glasgow Math. J. 19 (1978), no. 1, 13-26.

[10] Y. Ishigami, Linear Ramsey Numbers for Bounded-Degree Hypergrahps. Electronic Notes in Discrete Mathematics, 29 (2007), 47-51.

[11] P. Keevash, E. Long, J. Skokan, Cycle-complete Ramsey numbers, International Mathematics Research Notices, rnz119 (2019), https://doi.org/10.1093/imrn/ rnz119.

[12] B. Nagle, S. Olsen, V. Rödl, M. Schacht, On the Ramsey number of sparse 3-graphs, Graphs and Combinatorics, 24(3) (2008), 205-228.

[13] V. Nikiforov, C.C. Rousseau, Ramsey goodness and beyond., Combinatorica 29(2) (2009), 227-262.

[14] T. Kővári, V. T. Sós, P. Turán, On a problem of K. Zarankiewicz, Colloquium Math. 3, (1954). 50-57.

[15] F. P. Ramsey, On a Problem of Formal Logic, Proc. London Math. Soc. (2) 30 (1929), no. 4, 264-286.

[16] Y. Zhao, AIM workshop on Graph Ramsey Theory Problem Session, https://www . overleaf.com/read/mnvcscjjysvg. 